\title{
Evaluation of the Biosynthesized Silver Nanoparticles" Effects on Biofilm Formation
}

\author{
*11Nehia N. Hussein, ${ }^{1}$ Mina M. Khadum
}

${ }^{1}$ Biotechnology Division, Departments of Applied Sciences, University of Technology, Iraq

\section{Article information}

\section{Article history:}

Received: April, 07, 2021

Accepted: April 24, 2021

Available online: April, 26, 2021

Keywords:

Pathogenic

Microbes

Streptococcus

pyogenes

Virulence factor

Correspondence:

Nehia N. Hussein

100103@ uotechnology.edu .iq

\begin{abstract}
In this study 50 isolates were obtained from the Baghdad teaching city medicine laboratories, from wounds and burns. Isolates were identified exercise VITEK 2 system (Biomerieux). Streptococcus pyogenes isolate was used to create the biosynthesize of silver nanoparticles" against some pathogenic microbes such as Pseudomonas aeruginosa, Staphylococcus aureus, Escherichia coli, and Candida albicans. Evaluation of the effect of the created biosynthesis silver nanoparticles" (AgNPs) by Streptococcus pyogenes on the biofilm formation by various human pathogenic bacteria. Biosynthesis of AgNPs was characterized by ultraviolet-visible absorption spectroscopy, the observation of color change of the experimental samples in the presence of $1 \mathrm{mM} \mathrm{AgNO}_{3}$ at $410 \mathrm{~nm}$. A color change from pale yellow to slightly brown occurred for bacterial supernatant within 24 hours of incubation in the presence of light Scanning electron microscope (SEM), the biosynthesis silver nanoparticles" are predominately circular fit as a fiddle having a smooth surface and very much scattered with close minimal game plan, X-ray diffraction (XRD). The. The normal molecule size was determined by Debye-Scherer equation and its evaluation was roughly $6.43 \mathrm{~nm}$. The normal molecule size was determined by DebyeScherer equation and its evaluation was roughly $6.43 \mathrm{~nm}$. The importance of this work lies in the possibility of synthesizing the silver nanoparticles" using these bacteria, which are considered as types of fastidious bacteria. As far as the researcher's knowledge is concerned, this is study is the first of its kind in Iraq.
\end{abstract}

DOI: 10.53293/jasn.2021.11019, Department of Applied Science, University of Technology

This is an open access article under the CC BY 4.0 license.

\section{Introduction}

Biofilms have great importance for public health due to the main role in certain infectious diseases, also the importance in a variety of medical device-related infections. Therefore, there is a need to develop novel, effective and specific antimicrobial substances, which can be utilized to diminish the biofilm associated pathogenicity in hospital and other public spaces [1]. Original bacterial activities base initiative the set-far and presentation of biofilms, beyond stall assemblage or demise, nutrient acquisition, waste product accumulation, motility mechanisms and exopolysaccharide synthesis [2]. Biofilms represent bacterial communities deep-seated in self-delivered extracellular polymeric matrix that is connected to a surface. The microbial general public extensive a biofilm duff be thankful up of virtuous or multiple bacterial species. Bacteria have biofilms on unusual surfaces such as direct nautical systems, prime pipes, living tissues, indwelling medical devices and implants [3]. Biofilms are framed and may unescorted alteration the size and shape. A naturally expansively-ripened biofilm comprises an EPS design, 
which intercedes cell-cell correspondence (i.e. band sensing), loads the microorganisms advantaged the biofilm, and secures the creature unfamiliar natural pressure, particularly from anti-infection agents and cleansers. A positively fit biofilm forever has a Baroque finances duct in which oxygen, nutrients, and remodeling in turn relevant fitments derriere be metabolic wastes and transported and cell debris can be expelled [4]. The shapes sizes, and aspects of biofilms are joined wide bring forth of microbes, come installation, and environmental elements. Ignoring the increased familiarity of biofilm designate, inhibiting and excision microbial biofilms stay a difficult undertaking. Bacteria heart biofilm conduct significantly uniquely in contrast to free-living cells. Microbial biofilms mastery different antitoxin talents, such as adding to real surfaces, proclaim of French history impedance genes preferred biofilms, assurance of microscopic organisms from phagocytic murdering, and blockage of anti-toxin dissemination [5-8]. In this research, S. pyogenes bacteria were selected for the biosynthesis of silver nanoparticles". S. pyogenes is a $\mathrm{G}+\mathrm{ve}$, non-motile, facultative anaerobic and non-spore-forming bacteria. Streptococci showed in pairs or chains of varying length. Growing to a varying thermal temperature ranging between $22-40{ }^{\circ} \mathrm{C}$, weak growth in the quarters AGRO normal and improved by including serum or blood [9]. The extracellular synthesis of silver nanoparticles' using bacterial species acts to be acceptable to many applications [10]. And to improve new effective antimicrobial agents that overcome the MDR microorganisms [11]. Therefore, the present study has been designed to the biosynthesis of silver nanoparticles' using S. pyogenes bacteria to investigate the efficacy of the biosynthesized silver nanoparticles' in inhibiting the biofilm formation.

\section{Materials and Methods:}

\subsection{Collection and identification of microbial isolates:}

All (50) isolates were obtained from the Baghdad teaching city medicine laboratories, from wounds and burns. Isolates were identified exercise VITEK 2 system (Biomerieux). S. pyogenes isolate was used to create the biosynthesize of silver nanoparticles' against some pathogenic microbes such as P. aeruginosa, S. aureus, E. coli, and C. albicans. The bacterial isolates were preserved on nutrient agar. While yeast was preserved on Sabouraud dextrose agar (SDA) [12, 13].

\subsection{Biosynthesis of silver nanoparticles' :}

The growing S. pyogenes strains were freshly injected on Muller-Hinton broth and incubated at $37{ }^{\circ} \mathrm{C}$ for $24 \mathrm{~h}$. The culture was centrifuged at $14,000 \mathrm{rpm}$ for 10 minutes, takes the supernatant and added separately to the reaction container containing a silver nitrate concentration of 3-10 $(1 \% \mathrm{v} / \mathrm{v})$. The interaction between this supernatant and $\mathrm{Ag}^{+}$ions was carried out in bright status for $24 \mathrm{~h} \mathrm{[14].}$

\subsection{Characterization of biosynthesis silver nanoparticles':}

Silver nanoparticles' were construed using an Ultraviolet -Visible spectrophotometer. Color change modification of the response reaction blends was observed, through UV estimation an obvious response blend range, after intermittently weakening the response blend with distilled water, then estimated by UV-Vis spectrophotometer. For portrayal of the size and morphological of nanoparticles' scanning electron microscope instrument (SEM) investigation were utilized. The examination is being conducted by planning of slides by including little drops of suspension of bio combination nanoparticles' on slides, left to dry and then construed by SEM. Several drops of nanoparticle solution were placed on the surface of a glass slide and dried up to form a thin $0.5 \mathrm{~mm}$ thick layer. Then, they were examined using an X-ray diffractometer by casting $\mathrm{Cu} \mathrm{K \alpha}$ over the model to be measured at different angles from $20^{\circ}$ to $60^{\circ}$ and a wavelength of 1.5406 , and measured the results. The Debye-Scherer formula was applied to obtain the size of the synthesized nanoparticles' [15].

\section{Antibiofilm activity of silver nanoparticles': 3.1 Qualitative detection:}

A loopful of test organisms was inoculated in $10 \mathrm{~mL}$ of trypticase soy broth with $1 \%$ glucose in test tubes. The tubes were incubated at $37{ }^{\circ} \mathrm{C}$ for $24 \mathrm{~h}$. and $1 \mathrm{ml}$ of bacterial supernatant concentrations $(20,40,60,80$ and $100 \mu \mathrm{g} / \mathrm{ml})$, nanoparticles' and biosynthesized silver nanoparticle $(20,40,60,80$ and $100 \mu \mathrm{g} / \mathrm{ml})$ for each tube. After incubation, tubes were decanted and washed with phosphate buffer saline $(\mathrm{pH} \mathrm{7.3)}$ and dried. Tubes were then stained with crystal violet $(0.1 \%)$. Excess stain was washed with deionized water. Tubes were dried in inverted position. The scoring for tube method was done according to the results of the control strains. Biofilm formation was considered 
positive when a visible film lined the wall and the bottom of the tube. The amount of biofilm formed was scored as 1-weak/none, 2-moderate and 3-high/strong. The experiment was performed in triplicate and repeated three times [16].

\subsection{Quantitative detection:}

Tissue culture plate assay used by a lapful of microbes was injected in $10 \mathrm{~mL}$ of TSB in tubes. Transfer $100 \mu 1$ of the bacterial farm to the tissue culture plate; add $100 \mu \mathrm{l}$ of bacterial supernatant concentrations two- fold from supernatant and silver nanoparticles', except for positive and negative control wells. Add $200 \mu 1$ of sterile bacteria and incubate at $37{ }^{\circ} \mathrm{C}$ for 24 hours, then remove the contents of the wells and wash 3 times with phosphate solution. The wells were stained with crystal violet at $0.1 \%$ for 30 minutes, after which it were washed wells with distilled water and dried, $200 \mu 1$ of $95 \%$ ethanol was added to the wells [17].

\section{Results and discussion:}

\subsection{Biosynthesis of silver nanoparticles':}

The biosynthesis of silver nanoparticles' using supernatant was investigated primarily through the observation of color change of the experimental samples in the presence of $1 \mathrm{mM} \mathrm{AgNO}_{3}$. A color change from pale yellow to slightly brown occurred for bacterial supernatant within $24 \mathrm{~h}$ of incubation in the presence of light and is shown in (figure 1). This suggests the color change observed in the bacterial biomass and the supernatant sample were due to the formation of silver nanoparticles'. Changing in the color observed for the extracellular samples and further confirmed by UV-V spectral analysis as part of primary confirmation. Silver nanoparticles' are known to have an intense absorption peak in UV absorption spectra due to their surface Plasmon excitation. Figure (2) demonstrates the absorbance peak of synthesized AgNPs at different time interims which were finished by UV-vis spectrometer. The peak focused at $410 \mathrm{~nm}$, which is related to the absorbance of AgNPs.

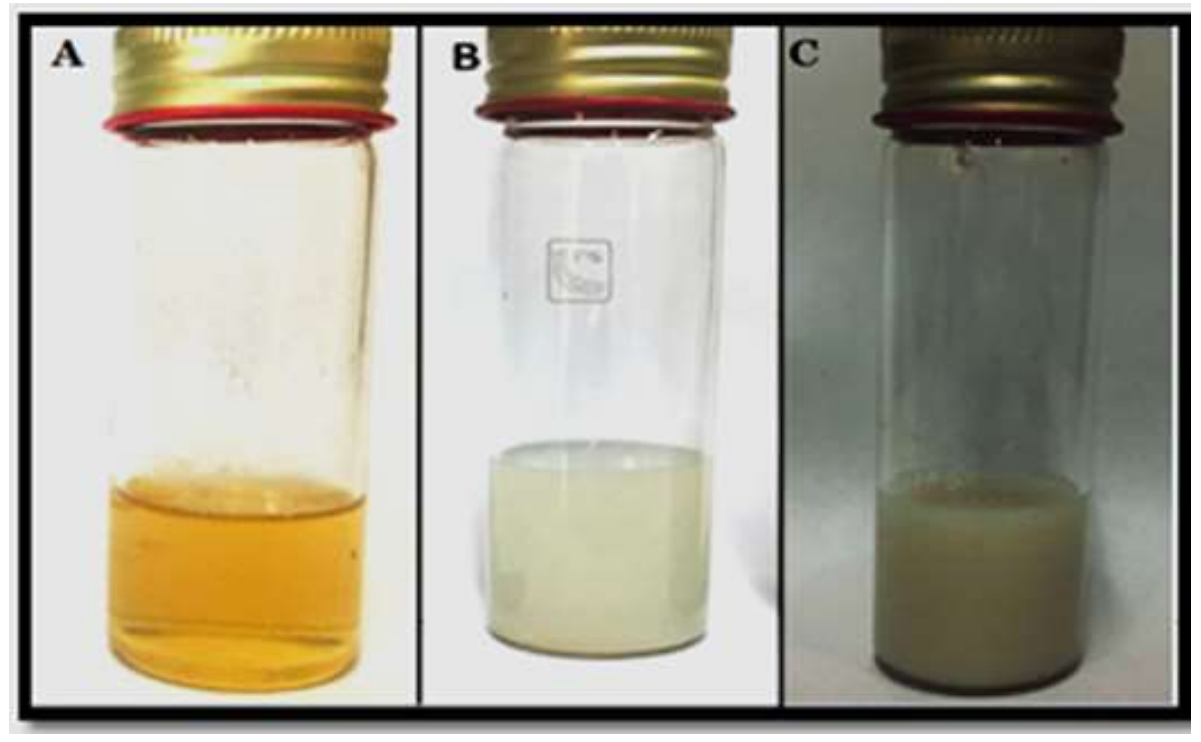

Figure 1. Synthesis of biosynthesized silver nanoparticles', A (at zero time), B (when adding silver nitrate to the supernatant of $S$. pyogenes bacteria), and C (Silver nanoparticles' after 24hrs). 


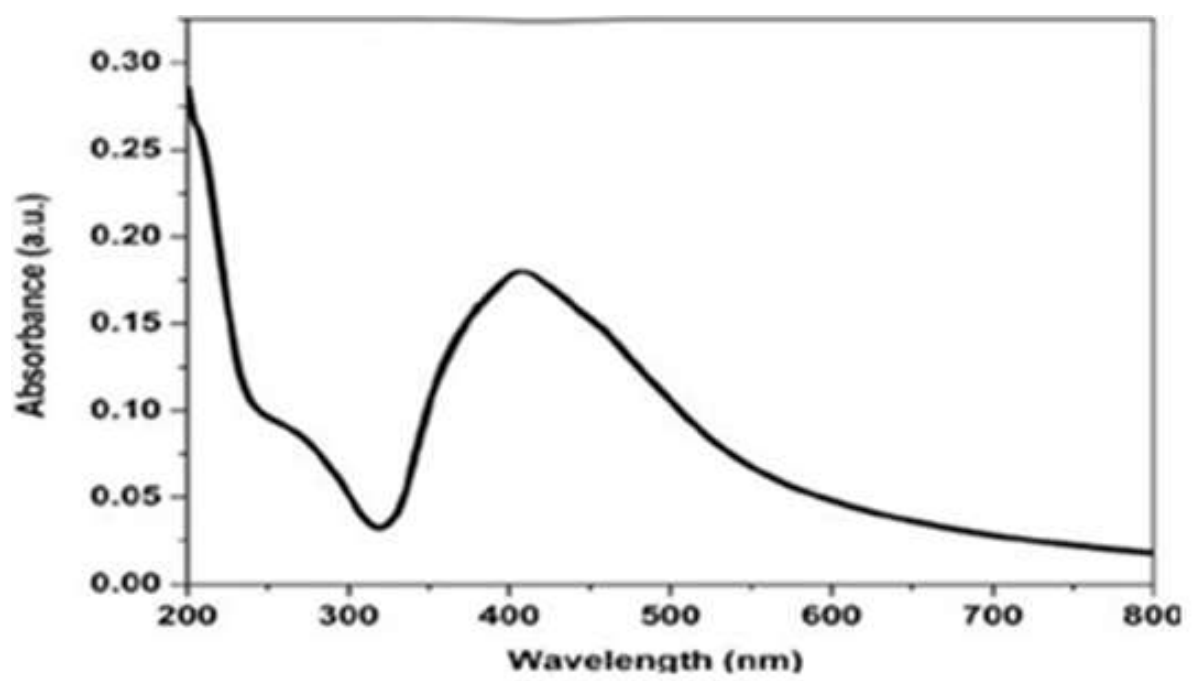

Figure 2. UV-Visible Spectrophotometer for biosynthesized silver nanoparticles'.

Figure (3) demonstrates the SEM pictures of the equivalent synthesized silver nanoparticles' sample. SEM pictures demonstrated that a large portion of the silver nanoparticles' is predominately circular fit as a fiddle having a smooth surface and very much scattered with a close minimal game plan. The normal molecule size was found within the range $(95.88-60.64 \mathrm{~nm})$.

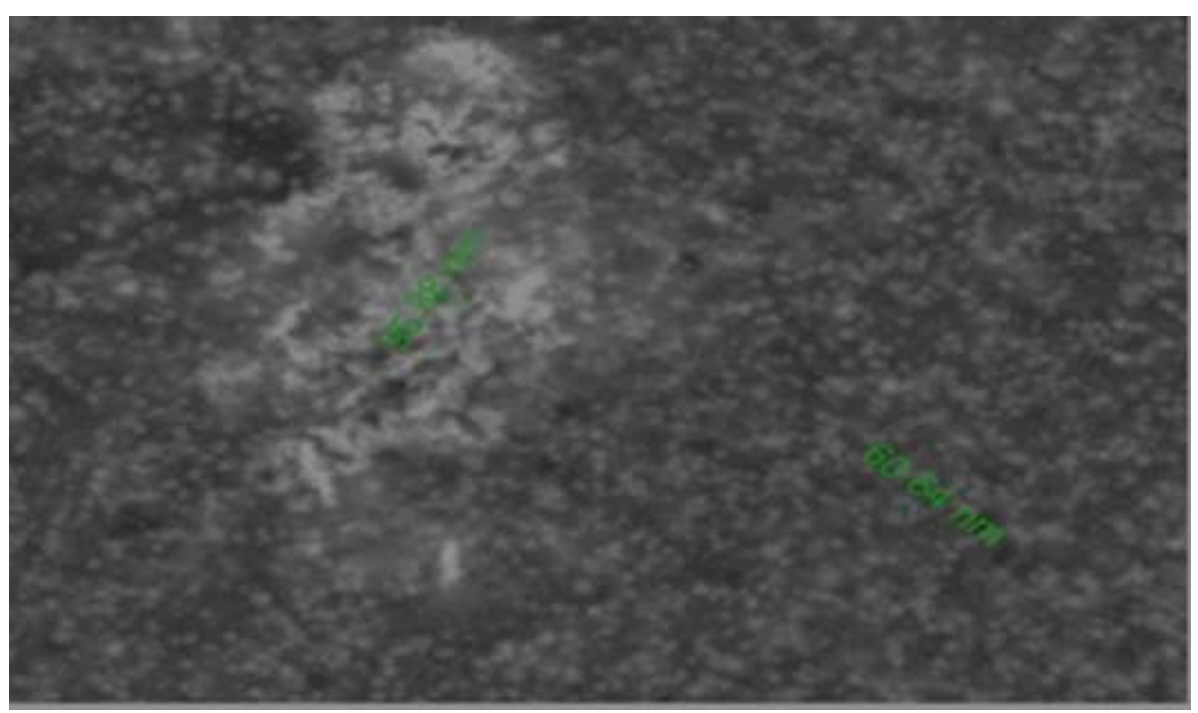

Figure 3. Characterization of nanoparticles' using SEM.

The normal molecule size was determined by the Debye-Scherer equation where full width at half most extreme (FWHM) information was utilized [18]. The normal molecule size evaluated was roughly $6.43 \mathrm{~nm}$. Debye-Scherer equation is: $\mathrm{D}=\mathrm{K} \lambda / \beta \cos$, where, $\tau$ is referring to the size of the molecule, $\mathrm{K}$ is referring to a dimensionless shape factor, $\lambda$ is the X-ray wavelength, $\beta$ is the line widening at a large portion of the greatest force (FWHM), $\theta$ is the Bragg edge (in degrees). Through the X-Ray Diffraction process, the formation of nano-silver at the angle $2 \theta$ was shown in degrees $(29.39,32.21$ and 31.77). By applying Scherrer equation, the calculated nanoparticles' were 30.3 nanometers. 


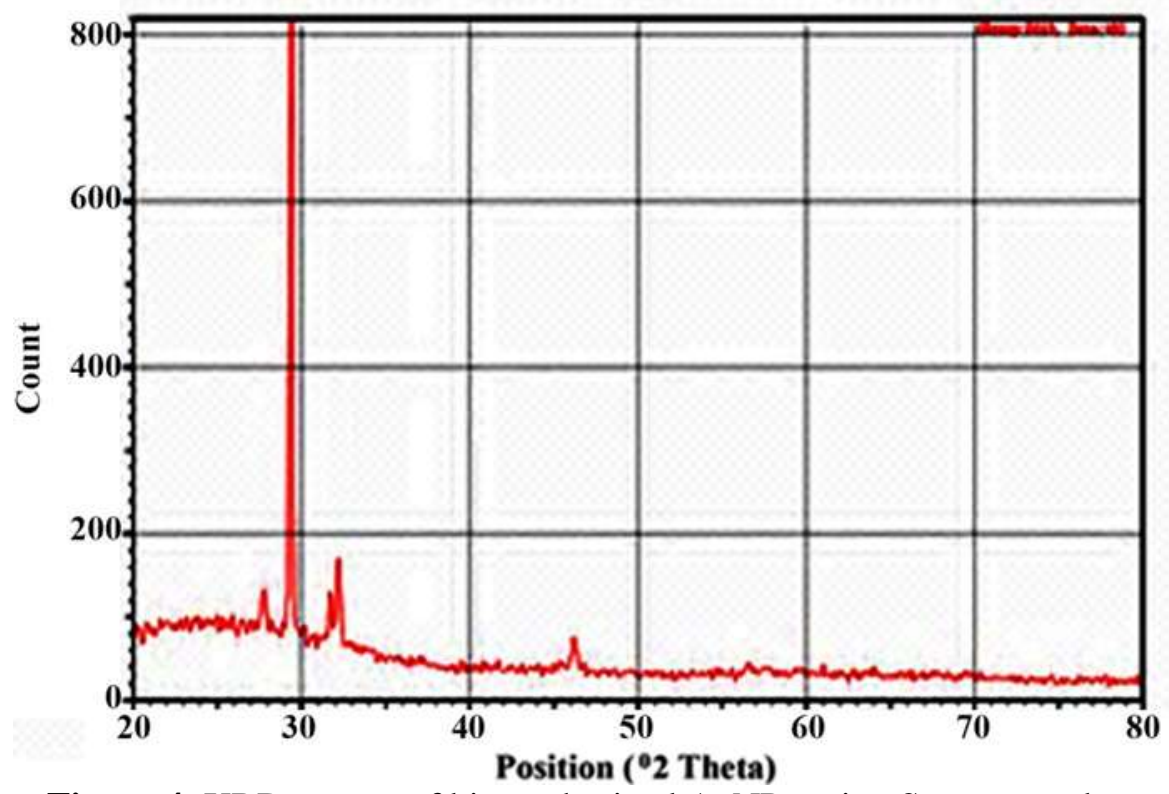

Figure 4. XRD pattern of biosynthesized AgNPs using S.pyogenes bacterium.

\subsection{Determination of Biofilm inhibitory concentration (BIC):}

\subsubsection{Test tube assay for qualitative detection of biofilm formation:}

Results in Table (1), and Figure (5), All tested microorganisms showed their ability to form biofilm in the form of film lined the wall and bottom of tubes in the tube method without treated by the nanoparticles'. Both the E. coli, and yeast C.albicans recorded a high ability to biofilm formation, while both the S. aureus, P. aeruginosa bacteria gave a moderate ability to biofilm formation. But when treated with AgNPs this ability was prevented and removed in all isolates. AgNPs may be altered gene the expression relating to biofilm formation, as consequence they effect on microcolony formation and biofilm maturation. This lead to AgNPs could be used for prevention and treatment of Biofilm-related infections [19]. The antibiofilm activity of AgNPs was observed less effective against G+ve and Gve microbes.

Table (1) Biofilm activity using test tube method for different microorganisms

\begin{tabular}{|c|c|c|}
\hline Isolate name & $\begin{array}{c}\text { Bacterial } \\
\text { Supernatant } \\
\text { Without AgNPs }\end{array}$ & $\begin{array}{c}\text { Biosynthesis AgNPs } \\
\text { by bacterial } \\
\text { supernatant }\end{array}$ \\
\hline E.coli & Strong & None \\
\hline S.aureus & Moderate & None \\
\hline P.aeruginosa & Moderate & None \\
\hline C.albicans & Strong & None \\
\hline
\end{tabular}



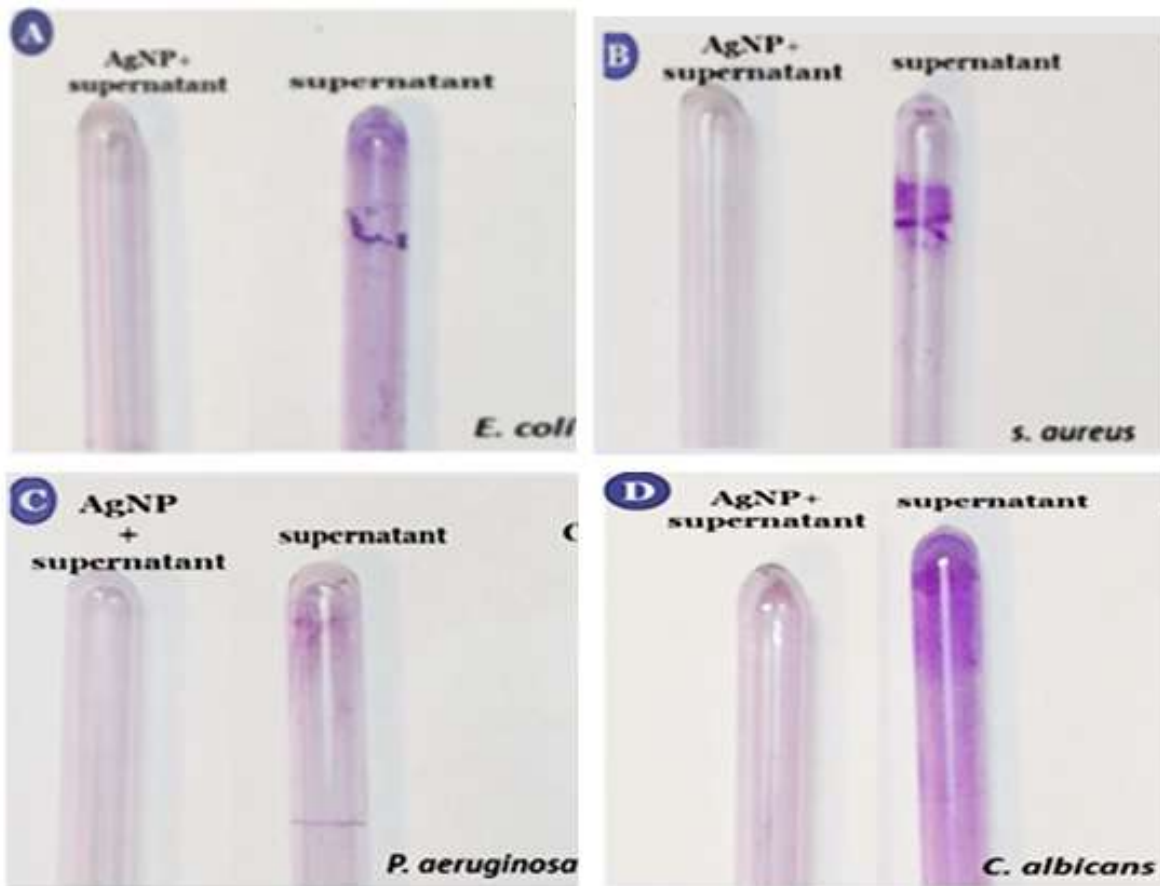

Figure 5. Qualitative detection of inhibition of the biofilm formation using tubes methods. (Supernatant) test tube refer to supernatant only without AgNPs (before treatment), (AgNPs + Supernatant) test tube refer to AgNPs synthesized by supernatant (after treatment).

\subsubsection{Tissue culture plate method for quantitative detection of biofilm formation:}

The biofilm detection method using the tissue culture plate made of polystyrene showed a gradient in the formation of the biofilm according to the concentration of the materials used. The amount of pigment that stained the negative control wells was very small, while the positive control wells were strongly pigmented, indicating the presence of dense biofilm, as for the filtrate treated pits, the biofilm formation was moderate and weak respectively, while the mixture treated wells produced a very weak biofilm, while the silver nanoparticles' showed an effect similar to the effect of bacterial filtration, and this indicates an increase in the inhibitory activity of the biosynthesis silver nanoparticles'. (figure 6).

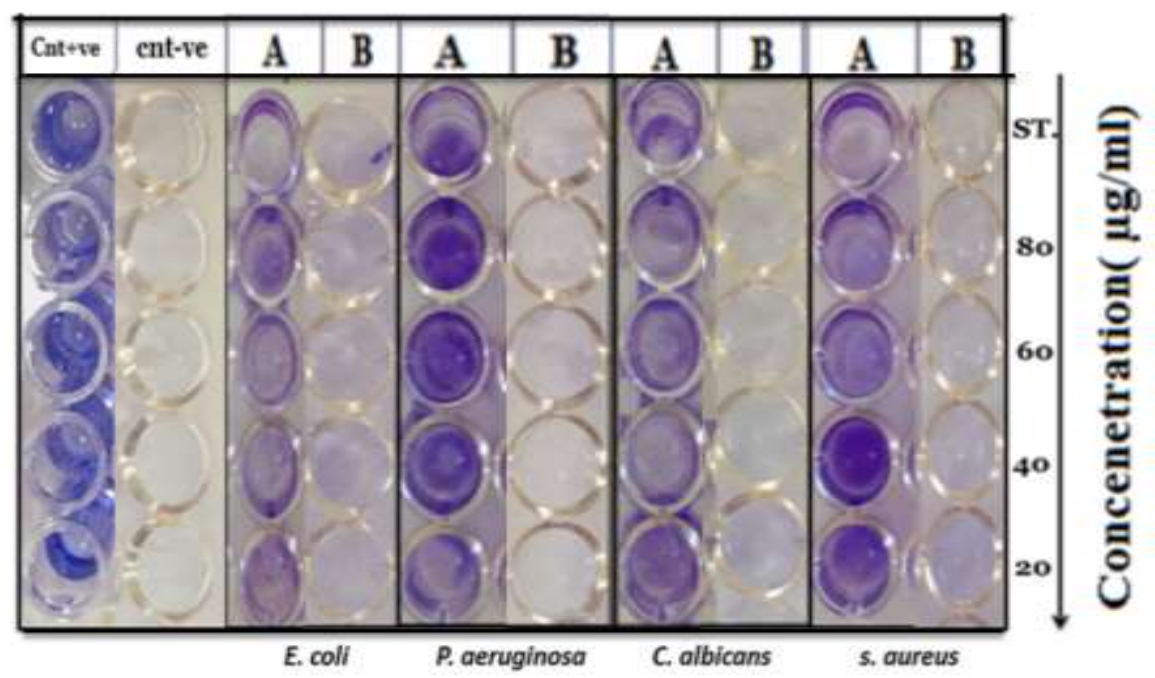

Figure 6. Quantitative detection of Inhibitory of biofilm formation by tissue culture plate method (A) Refer to bacterial supernatant only (before treatment), and (B) after treatment with biosynthesis silver nanoparticles' for each isolates. 
Silver ions weaken the biofilm formation in bacteria, after their transfer into the bacterial cell and their interaction with proteins and enzymes required for microbial adhesion, these results in a decrease in the activity of the biofilm [20]. Alternatively, they inhibit biofilm formation by inhibiting the formation of external polysaccharides. The mechanism of inhibiting the biofilm formation process by using nanoparticles' is done through penetration of those particles into the water channels that serve to transport nutrients through a layer of polysaccharides [21- 23]. Its small size helps it to penetrate the sugars layer and creates contact between it and the bacterial cells, thus inhibition of the biofilm formation process $[24,25]$. Exposure of the membrane biosynthesis to silver nanoparticles' reduces the membrane components (proteins, polysaccharides, lipid, phospholipid, nucleic acid), causing the membrane to gradually break down. The sugars in the biofilm are (rhamnose, mannose, galactose, glucose), which are present in high abundance, although xylose is present in the polysaccharides of some bacteria, but its presence is sometimes considered uncommon, these sugars play an important role in the formation of the biofilm. Increasing the concentration of nanoparticles' reduces the concentration of carbohydrates and proteins, which leads to weakening the membrane structure and thus facilitates the passage of drugs and treatments [26,27]. Bacterial cells thus get inhibition of the process of biofilm formation [28]. Biofilm-forming microbes can cause various diseases, and according to one of the reports presented by the National Institutes of Health and Centre of Disease Control, biofilmforming microbes are responsible for causing $65-80 \%$ of infections $[29,30]$. Thus, one of the efficient strategies to control the infections by these microbes is to use biofilm inhibitors. Various studies have indicated the effective role of NPs as biofilm inhibitors against target bacteria. One of our previous studies indicated the anti-biofilm and antiadhesion potentials of magnesium oxide nanoparticles' against drug-resistant bacteria [31, 32].

\section{Conclusions:}

Biofilms cause resistance too many antimicrobial agents. The results of biofilm produced on indwelling medical devices are recurrent, untreatable infections and failure of medical device. To overcome chronic and recurrent infections, it is important to detect biofilms of microorganisms, determine antibiofilm activity of agents against biofilm. In addition, to determine antibacterial activity of agents against biofilm embedded microorganism with the appropriate methods by clinical microbiologist and biofilm researcher microbiologist. Identification of genes involved in biofilm formation and measurement of gene expression as a result of antibiofilm and antibacterial activity of agents can be advantageous in biofilm studies. The results in this study recommended that the integration of silver with bacteria, there were considerable anti-biofilm impacts in all concentration of AgNPs against various human pathogenic bacteria by qualitative and quantitative methods.

\section{Acknowledgment:}

The authors would like to extend their sincere appreciation to the Department of Applied Science Laboratories, University of Technology, Baghdad, Iraq for its experimental assistance. No funding support by our institution was provided. The authors are responsible for their funding support.

Conflict of interest statement: There are no conflicts of interest regarding the publication of this manuscript.

\section{References:}

[1] K. Vasdev, S. Dewasthale, I. Mani. Microbial biofilm: current challenges in health care industry: Appl Biotechnol Bioeng.5 (3):160-164.2018.

[2] S. Haussler, C .Fuqua, Biofilms 2012: New discoveries and significant wrinkles in a dynamic field: J Bacteriol, 195; 2947-2958.2013.

[3] R.M. Donlan, Biofilms: microbial life on surfaces: Emerg Infect Dis., 8: 881-890.2002.

[4] J.B. Kaplan. Biofilm dispersal: mechanisms, clinical implications, and potential therapeutic uses: J. Dent. Res, 89; 205-18.2010.

[5] M. Klausen, A. Aaes-Jorgensen, S. Molin, T. Tolker-Nielsen, Involvement of bacterial migration in the development of complex multicellular structures in Pseudomonas aeruginosa biofilms: Mol Microbial, 50:618.2003.

[6] T.F.C. Mah, G.A. Toole, Mechanisms of biofilm resistance to antimicrobial agents: TRENDS MICROBIOL, 9: 34-9.2001.

[7] R. Singh, P. Ray, A. Das, M.Sharma, Penetration of antibiotics through Staphylococcus aureus and Staphylococcus epidermidis biofilms: J Antimicrob Chemother, 65:1955-8.2010.

[8] A.J. Huh, Kwon YJ, "Nanoantibiotics": a new paradigm for treating infectious diseases using nanomaterials in 
the antibiotics resistant era: J Control Release, 156:128-45.2011

[9] J.McFadden, Biochemical test for identification of medical bacteria "3rd edition ": The Willians and Wilkins. Baltinor, USA. 2000.

[10] p. Mohanpuria , Rana, N.K. , Yadav, S.K. ;Biosynthesis ofnanoparticles': technological concepts and future applications; Journal of Nanoparticle Research; 2008; vol. 10, no. 3, pp. 507-517.

[11] S. Karthick, Namasivayam, R., Aroma,R. ,Manikanta, M. , Gopinath,P. ,.Francis ,A.L ; Evaluation of enzyme activity inhibition of biogenic silver nanoparticles' against microbial extracellular enzymes ;International Journal of PharmTech Research .2016; Vol.9, No.2, pp 40-47.

[12] N.H.Nehia, H.M.Amen.Detection of the antibacterial activity of AGNPS Biosynthesis by Pseudomonas aeruginosa, Iraqi J. Agric. Sci, 50(2): 617-625.2019.

[13] D. M. Saria, N.H.Nehia,A.H. Buthynia. Effect of Antibiotics with Laser Radiation on Growth of Two Pathogenic Bacteria Species: Engineering and Technology Journal, 30(7).2012.

[14] G.Caroling, S.K.Tiwari,A.M. Ranjitham, , R. Suja, , Biosynthesis of silvernanoparticles' using aqueous broccoli extract-characterization and study ofantimicrobial cytotoxic effects: Asian J. Pharm. Clin. Res, 6: 165-172.2013.

[15] A.A.Reem, N.H.Nehia,F.A. Khlida, Detection of the antimicrobial activity of AGNPS by Quercus infectoria plant: Plant Arch, 20(1):579-584.2020.

[16] Christensen GD, Simpson WA, Anglen JO, Gainor BJ. Methods for evaluating attached bacteria and biofilms. In: An YH, Friedman RJ, editors. Handbook of Bacterial Adhesion: Principles Methods, and Applications. Totowa: Humana Press; 2000. pp. 213-233

[17] Q.Hu, X.Han, X.Zhou, S.Ding, C.Ding, S.Yu , Characterization of biofilm formation by Riemerella anatipestifer. Vet. Microbiol, 144; 429-436.2010.

[18] V.Das, R.Thomas, , R.Varghese, , E.V.Soniya, J.Mathew, and et al," Extracellular synthesis of silver nanoparticles' by the Bacillus strain CS 11 isolated from industrialized area ", 3 Biotech, 4: 121-126.2014.

[19] Abdulhassan,A.J.;.Effect of Silver and Titanium Nanoparticles' Synthesized by Lactobacillus as Antimicrobial ,Antioxidant and Some Physiological Parameters .Master Thesis .University of Kufa ,Faculty of Science Iraq.2016;::86-93.

[20] S.Gavanji, S.S.Sayedipour, ,M. Doostmhammadi , and B. Larki, , "The effect of different concentration of silver nanoparticles' on enzyme activity and liver tissue of adult male wistar rats in - vivo condition" .IJSRK,4(2):182-188.2014.

[21] F.Zhang, I.L.Mao, Z.Wei, and J.Zhang, "Bactericidal effect of nano-silver against E. faecalis growing in multispecies biofilm". Journal of Practical Stomatology, 33(3): 397-400.2017.

[22] A.Mirhashemi, A.Bahador, M.Kassaee, G.Daryakenari, M.Ahmad-Akhoundi, and A. Sodagar, "Antimicrobial effect of nano-zinc oxide and nano-chitosan particles in dental composite used in orthodontics". Journal of Medical Bacteriology, 2(3-4): 1-10.2013.

[23] A.J. Haider, A.D. Thamir, D.S. Ahmed, M.R. Mohammad,Deposition of silver nanoperticles on multiwalled carbon nanotubes by chemical reduction process and their antimicrobial effects. AIP Conference Proceedings 1758 (1), 030003, 2016

[24] A.J. Haider, A.J. Mohammed, S.S. Shaker, K.Z. Yahya, M.J. Haider, Sensing characteristics of nanostructured SnO2 thin films as glucose sensor. Energy Procedia 119, 473-481, 2017.

[25] D.Wu, W.Fan, A.Kishen, J.L.Gutmann, and B.Fan, "Evaluation of the antibacterial efficacy of silver nanoparticles' against Enterococcus faecalis biofilm". J. Endod., 40(2): 285-290.2014.

[26] A.J. Haider, R.A. Al-Rsool, M.J. Haider,Morphlogical and structural prpperties of cathode coumpound material for lithium -ion battery .. Plasmonics 13 (5), 1649-1657, 2018

[27] P.Cheng, J.Wang, and T. Liu, "Effects of nitrogen source and nitrogen supply model on the growth and hydrocarbon accumulation of immobilized biofilm cultivation of B. braunii". Bioresour. Technol, 16(6): 527 533.2014.

[28] K.S.Siddiqi, A.Husen, "Fabrication of metal nanoparticles' from fungi and metal salts: scope and application. Nano Res Lett, 11:98. 2016.

[29] N. Deka, Comparison of Tissue Culture plate method, Tube Method and Congo Red Agar Method for the detection of biofilm formation by Coagulase Negative Staphylococcus isolated from Non-clinical Isolates". Int. J. Curr. Microbiol. App. Sci, 3(10):810-815. 2014.

[30] M.W.Jamal S.Ahmad, Andleeb et al., "Bacterial biofilm and associated infections," Journal of the Chinese 
Medical Association, vol. 81, no. 1, pp. 7-11, 2018.

[31] S. Hayat , M.H. Muzammil, Rasool et al., "In vitro antibiofilm and anti-adhesion effects of magnesium oxide nanoparticles' against antibiotic resistant bacteria," Microbiology and Immunology, vol. 62, no. 4, pp. 211-220, 2018.

[32] R.M.D. A.A. Atiyah, A. J. Haider, Cytotoxicity properties of functionalized carbon nanotubes on pathogenic bacteria. IET Nanobiotechnology, 2019. 